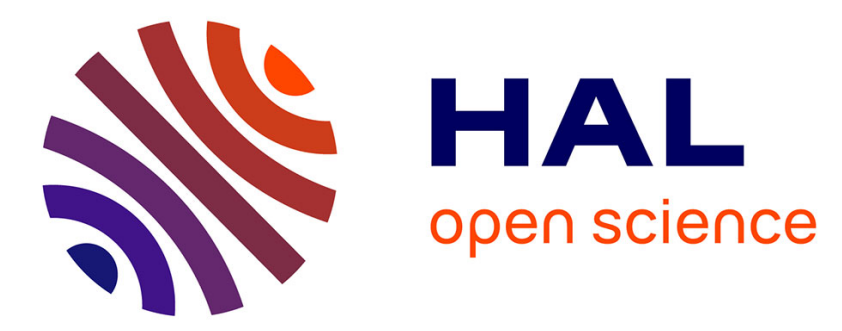

\title{
The use of superconducting junctions in magnetometry
}

J. Clarke

\section{To cite this version:}

J. Clarke. The use of superconducting junctions in magnetometry. Revue de Physique Appliquée, 1970, 5 (1), pp.32-36. 10.1051/rphysap:019700050103200 . jpa-00243370

\section{HAL Id: jpa-00243370 https://hal.science/jpa-00243370}

Submitted on 1 Jan 1970

HAL is a multi-disciplinary open access archive for the deposit and dissemination of scientific research documents, whether they are published or not. The documents may come from teaching and research institutions in France or abroad, or from public or private research centers.
L'archive ouverte pluridisciplinaire HAL, est destinée au dépôt et à la diffusion de documents scientifiques de niveau recherche, publiés ou non, émanant des établissements d'enseignement et de recherche français ou étrangers, des laboratoires publics ou privés. 


\title{
THE USE OF SUPERGONDUGTING JUNGTIONS IN MAGNETOMETRY
}

\author{
By J. CLARKE, \\ Department of Physics, University of California and Inorganic Materials Research Division, \\ Lawrence Radiation Laboratory, Berkeley, California 94720.
}

Introduction. - This paper is divided into two parts. The first describes a superconducting device (the Slug) which has proved useful in the measurement of electrical currents down to $30 \mathrm{nA}$, voltages down to $10^{-15} \mathrm{~V}$ and magnetic fields down to $10^{-8}$ gauss. In the second part, we discuss the properties of a type of Josephson junction in which the conventional oxide barrier is replaced by a copper barrier.

I. The Slug as a Magnetometer. - 1. Principle of THE MAGnetometer. - The principle of the magnetometer is extremely simple. Consider a superconducting ring of radius $R$ and inductance $L$. A superconducting galvanometer of negligible inductance is mounted on the ring so as to measure the current flowing in it. Let the smallest current which can be detected by the galvanometer be $\Delta I$. We assume for simplicity that initially the applied field is zero so that no flux is trapped by the superconducting ring. If we now apply a magnetic field at right angles to the plane of the ring, flux quantization demands that a circulating supercurrent be established so as to maintain the fluxoid at its original value. The magnitude of this supercurrent, measured by the galvanometer, will be $I=\Phi / L$, where $\Phi$ is the magnetic flux applied to the ring. The smallest detectable change in flux is clearly given by :

$$
\Delta \Phi=L \Delta I .
$$

Notice that as we increase the area of the ring, and therefore its inductance, the sensitivity of the magnetometer to magnetic flux decreases. On the other hand, the sensitivity of the detector to magnetic field (flux/ area) increases. The magnetic field resolution is given by :

$$
\Delta H=\frac{\Delta \Phi}{\pi R^{2}}=\frac{L \Delta I}{\pi R^{2}} \sim \frac{\Delta I}{R},
$$

since inductance is roughly proportional to the radius of the ring. Thus the magnetometer may be made arbitrarily sensitive to a uniform magnetic field by simply increasing the area of the superconducting ring.

2. Principle of the Galvanometer. - We require a galvanometer which is superconducting, has a low self-inductance, and whose current sensitivity is as high as possible. A suitable device has been developed which makes use of the properties of two Josephson [1] junctions in parallel.

A Josephson junction consists of two superconductors separated by an insulating barrier of 10-20 $\AA$ in thickness. We may ascribe an order parameter to each superconductor of the form $\psi=|\psi| \mathrm{e}^{i \varphi}$, where $|\psi|^{2}$ represents the density of condensed pairs. In a Josephson junction, the phases of the two superconduc- tors are locked together across the barrier. If we pass a sufficiently small current through the junction, Cooper pairs are able to tunnel through the barrier without developing a voltage across it. The barrier is thus able to sustain a D.C. supercurrent but only up to a maximum value known as the critical current, $i_{\mathrm{c}}$. For values of current greater than $i_{\mathrm{e}}$, the time-independent phase coherence is broken and a finite voltage appears across the junction. For values of current less than $i_{\mathrm{c}}$, the current is related to the phase difference across the barrier, $\Delta \varphi$, by the relation [1] :

$$
i=i_{\mathrm{c}} \sin (\Delta \varphi)
$$

If we have two identical junctions connected in parallel as shown in figure 1 , the total critical current is not necessarily the sum of the two individual critical currents. Jaklevic et al. [2] demonstrated that a magnetic field applied at right angles to the double

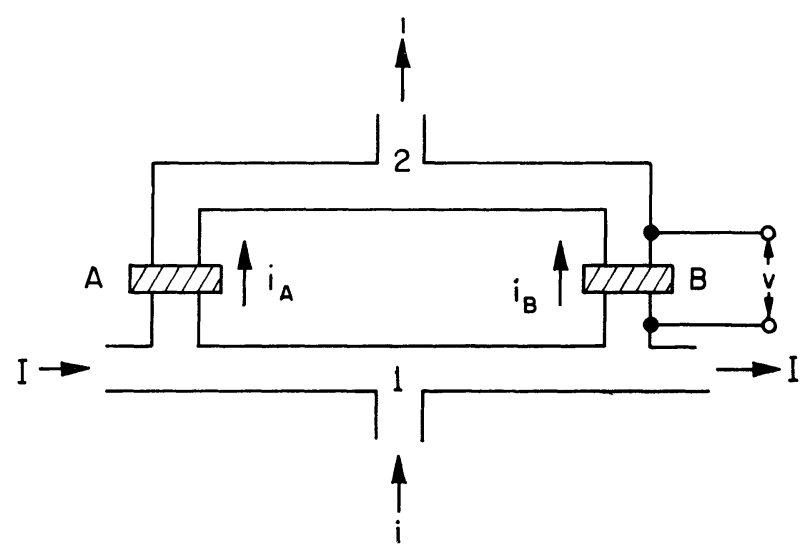

FIG. 1. - Double junction.

junction ring modulates the critical current periodically, the period being the flux quantum, $\Phi_{0}$, approximately $2 \times 10^{-7}$ gauss. $\mathrm{cm}^{2}$. A detailed description of the mechanism of this modulation is given (for example) elsewhere in this proceedings by $\mathrm{R}$. de Bruyn Ouboter and A. Th. A. M. de Waele.

Suppose that we pass a current I along one of the superconductors forming the double junction, as indicated in figure 1 . The current will link magnetic flux to the circuit and modulate the critical current. Let $I_{0}$ be the current required to generate one flux quantum in the ring, that is, to drive the critical current through one oscillation. Then the system may be used as a digital ammeter with a resolution $I_{0}$. However, in practice it is relatively easy to detect a change in critical current corresponding to $0.1 \%$ of one period. We may therefore measure a current change of $10^{-3} I_{0}$ although the measurement is no longer of a digital nature. 
3. A Practical Galvanometer. - Junctions have been made by forming a pellet of tin-lead solder, typically $8 \mathrm{~mm}$ long, on a length of niobium wire about $10^{-2} \mathrm{~mm}$ in diameter. The niobium has an oxide layer on its surface which prevents the solder making an intimate metallic contact with the wire. Instead the solder forms a tight mechanical contact around the oxide so that the junction consists of two superconductors separated by an insulator. A crosssection is shown in figure 2 . The characteristics of the device may be measured by means of the four-terminal network indicated.

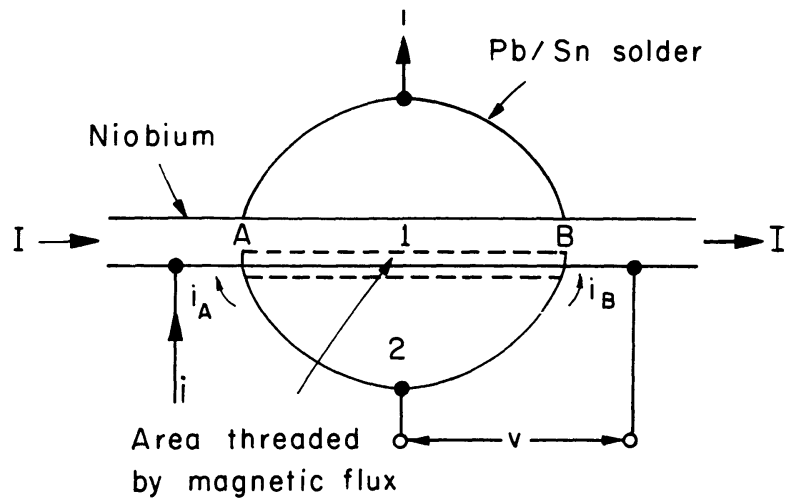

FIG. 2. $-C / S$ through Slug.

A typical junction has a resistance at room temperature of about $1 \Omega$. At liquid helium temperatures, these junctions exhibit some form of Josephson or weak-link behaviour with a critical current of the order of $1 \mathrm{~mA}$. A typical $i-V$ characteristic is shown in figure 3. One would expect the junction between wire and solder to be more or less continuous, although of varying thickness. However, it can be demonstrated by means of the following experiment that in

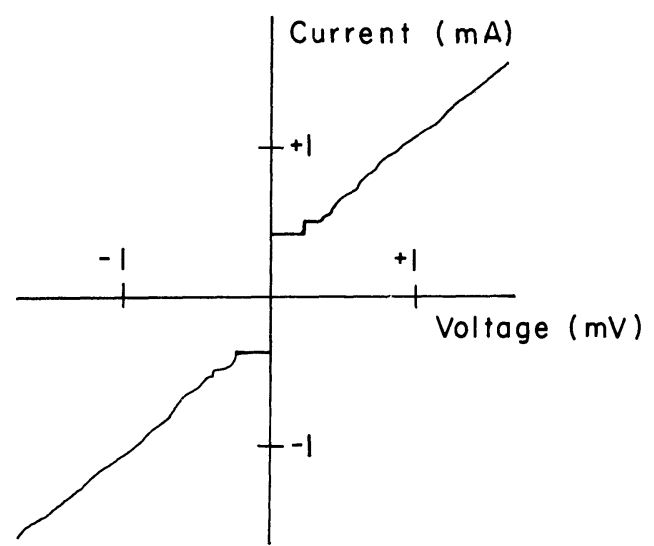

FIG. 3. - Typical $i-V$ characteristic of Slug.

fact only a small number of discrete junctions are formed. Let us measure the critical current $\left(i_{\mathrm{c}}\right)$ continuously and at the same time slowly increase a current $I$ along the niobium wire. It is found that the critical current is an oscillating function of $I$ with a typical period of $1 \mathrm{~mA}$.

A simple description of the modulation can be given as follows. Suppose that we have just two contacts within the device near the ends of the solder, labelled A and $\mathrm{B}$ in figure 2 . The current $I$ generates a field $2 I / r$ around the wire : this field enters the gap between wire and solder and also the penetration depths of both superconductors. We see that there is a clear analogy between the geometries of figures 1 and 2 . We can calculate the current change, $\Delta I$, required to generate one flux quantum in the enclosed area and thus cause the critical current to undergo one oscillation. Let $\lambda_{N b}$ and $\lambda_{s}$ be the penetration depths of niobium and solder respectively, $l$ the length of the specimen and $r$ the radius of the wire. Then we find :

$$
\Delta I=\frac{\Phi_{0} r}{2 l\left(\lambda_{N b}+\lambda_{s}\right)} .
$$

If we take $r \sim 5 \times 10^{-3} \mathrm{~cm}, l \sim 5 \times 10^{-1} \mathrm{~cm}$, $\lambda_{N b} \sim 500 \AA$ and $\lambda_{s} \sim 1,000 \AA$, we obtain :

$$
\Delta I \sim 700 \mu \mathrm{A} .
$$

This is in reasonable agreement with the observed period.

In general, more than two junctions may be formed. Two examples of the dependence of $i_{\mathrm{c}}$ upon $I$ are shown in figure 4 . In (a), the junction has just two junctions,

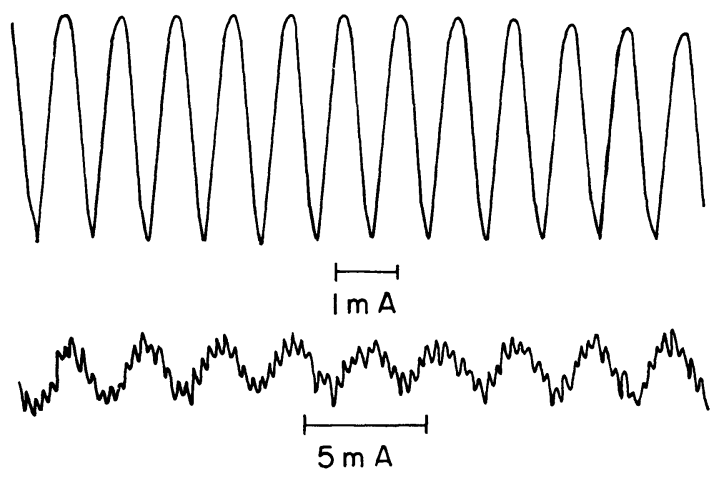

FIG. 4. $-i_{c}$ (vertical) against $I$ (horizontal) for two typical slugs.

whereas in (b), at least three junctions are present. However, the important feature for the present purpose is that a change in the current $I$ in the niobium wire produces a corresponding change in the critical current $i_{\mathrm{c}}$. Since the device is invariably used in a feedback circuit, the detailed structure of the oscillations is not important.

The exact details of the nature of the junctions are not known. However, a plausible explanation for the formation of discrete junctions is that the wire is microscopically very rough so that the solder, which does not wet the surface, makes a contact sufficiently thin to permit tunnelling at only a few points. From the fact that at least $10^{3}$ oscillations in critical current may be observed without any discernible diffraction envelope such as would be caused by junctions of finite width, we can deduce that the width of these contacts must be at least $10^{4}$ times smaller than the length of the solder pellet, that is, $1 \mu$.

Relatively little external circuitry is required for the galvanometer, which has become known as the SLUG (Superconducting Low-inductance Undulatory Galvanometer). The critical current has been measured by the following technique. A sinusoidal current sweep at about $20 \mathrm{kHz}$ was applied to the leads $(i)$ of figure 2. The sweep was confined to a region around the critical current so that the voltage which appeared across the junction as a function of time was a series of 
pulses, the leading edge being defined by the point in the cycle at which the critical current was exceeded and the trailing edge by the point at which the current returned to sub-critical values. These pulses have a mark-space ratio related to the fraction of the cycle during which there was zero voltage across the junction. After amplification, the pulses are integrated to produce an output voltage proportional to the critical current. By this technique, a current change of $10^{-7} \mathrm{~A}$ in the niobium wire can be readily detected. McWane et al. [4] have described an alternative method which uses a D.C. technique and Zych [5] an A.C. method which makes use of a lock-in detector.

It is not completely clear as to what limits the current resolution of a Slug. A change in the current flowing in the niobium wire gives rise to a comparable change in the critical current which (assuming a current source) is measured as a change in the voltage across the junction. Any noise generated in this resistance will of course limit the resolution; however, the Johnson noise expected in the junction in a bandwidth of $1 \mathrm{~Hz}$ is only about $10^{-11}$ volt. It appears more likely that noise picked up by the cryostat leads and transmitted into the junction is the limiting factor. By placing the cryostat in a shielded room, Goree (private communication) has found that the resolution of a Slug may be improved by at least an order of magnitude. The limit of resolution is then usually set by the input noise of the amplifier used to measure the voltage developed across the Slug. Rumbo and Wade (private communication) have effected further improvements by using a transformer and impedance match the Slug to the amplifier. A combination of these techniques would probably enable one to achieve a current resolution in the $10^{-9} \mathrm{~A}$ range.

4. Use of the Slug as a Magnetometer. - The Slug may be used as a magnetometer by simply connecting together the ends of the niobium wire so as to form a superconducting ring. W. S. Goree (private communication) has used this system with a ring diameter of $1.5 \mathrm{~cm}$ to achieve a sensitivity of $10^{-8}$ gauss with a time-constant of $1 \mathrm{~s}$. The corresponding current sensitivity was $3 \times 10^{-8} \mathrm{~A}$.

In practice, the magnetometer loop is best used as the sensor in a feedback circuit, as suggested in figure 5 .

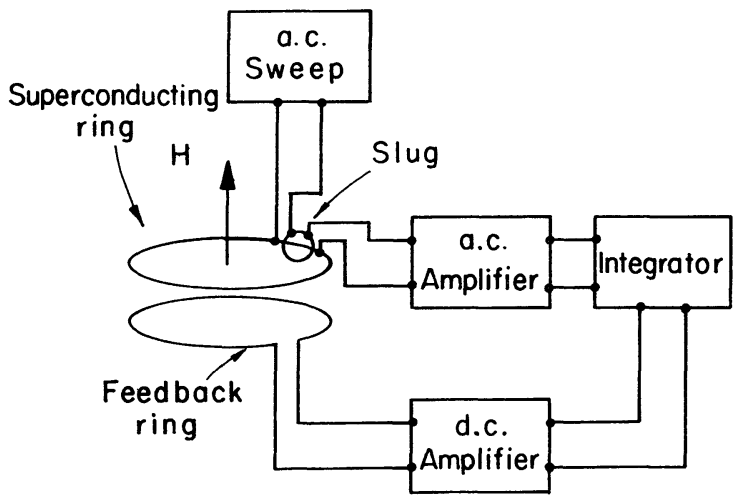

FIG. 5. - Feedback magnetometer.

The signal from the Slug is suitably amplified and fed back into a loop coupled to the superconducting ring so as to oppose the change in the applied field. This system may also be used as a sensitive magnetic field stabilizer.
5. Discussion. - The Slug used as a magnetometer has the advantage of being an extremely simple device. The electronic circuitry associated with it is also simple and with the aid of integrated circuits, may be made very compact. With the ring of about $2 \mathrm{~cm}^{2}$ described above, the magnetic field resolution is at least an order of magnitude less than that of the Squid developed by the Ford Group and that of the device described by Nisenoff elsewhere in these proceedings. However, as pointed out in (1), the present system could in principle be made arbitrarily sensitive by an appropriate increase in the area of the superconducting ring.

II. Junctions with Normal Metal Barriers. - 1. ThE Proximity Effect. - In a superconductor, the electrons are bound together in Cooper pairs by a weakly attractive interaction. The pair wave-function $\psi$ cannot be made to change abruptly in space but only over distances of the order of the Ginzburg-Landau coherence length $\xi_{s}(T)$, where $\xi_{s}(T)=\xi_{s}(0)(1-t)^{-1 / 2}$ and $\xi_{s}(0) \simeq \hbar v_{F} / k T_{\mathrm{c}}$. $\quad v_{F}$ is the Fermi velocity, $T_{\mathrm{c}}$ the transition temperature of the superconductor and $t=T / T_{\mathrm{c}} . \quad$ A typical value for $\xi_{\mathrm{s}}(0)$ is $500 \AA$.

Consider a superconductor (S) and a normal metal (N) which are in intimate electrical contact. Some of the paired electrons then diffuse into the normal metal while there is a corresponding depression of the amplitude of the superconducting order parameter near the interface. This phenomenon, known as the "proximity effect" [6], is a direct consequence of the finite coherence length, $\xi$, of the pair wave function : the function cannot fall abruptly to zero at the interface but must change relatively slowly, over a distance of the order of $\xi$. There is, therefore, a finite condensation amplitude in the normal metal near the interface. However, it is most important to realize that this wave function is evanescent, that is, it cannot propagate. To understand the significance of this remark, let us pass an electrical current across the interface of an NS system, assuming for simplicity that it is at $T=0$. We should then find that the resistance of the normal metal persists right up to the interface at which point the current becomes resistanceless. Despite the presence of the finite Cooper pair density, the current flows resistively throughout the normal region.

Upon what scale does the superconducting wave junction penetrate into the normal metal? Let us suppose that superconducting pairs are injected into the normal metal at a temperature $T$. The Fermi energy will be subject to fluctuations of magnitude roughly $k T$. The momenta of these electrons are then contained in a band :

$$
\Delta P \sim\left(\frac{k T}{E_{F}}\right) P_{F} \sim \frac{k T}{v_{F}} .
$$

By the uncertainty principle, the pairs will then decay with a characteristic length $\xi_{N}(T) \sim \hbar v_{F} / k T \sim 1 \mu$ in pure copper at $4{ }^{\circ} \mathrm{K}$. If the metal is "dirty", that is, $l \ll \xi_{N}$, the decay length becomes :

$$
\xi_{N}=\sqrt{\hbar v_{F} l / 6 \pi k T},
$$

where $l$ is the electronic mean free path. It may be shown [6] that the decay of the pair amplitude is approximately exponential, with a decay length $\xi_{N}$, that is, $\psi_{N} \propto \exp \left(-x / \xi_{N}\right)$. 
2. Supergondugtor-Normal Metal-SupercondugTOR JUNGTIONS. - Consider now a junction consisting of a normal metal sandwiched between two superconductors (SNS junction). The pair wave functions induced by the superconductors will decay into the normal metal but (in principle) overlap with some well-defined overlap energy. If this energy exceeds the thermal fluctuations to which the junction is subjected ( $\sim k T$, where $T$ may be $300{ }^{\circ} \mathrm{K}$ if the junction is connected by unfiltered leads to the outside world [1]), then the two superconductors will be coupled together. The barrier is now able to sustain a supercurrent and the SNS junction will behave in essentially the same way as the oxide junctions mentioned in I $(\S 2)$.

However, there are several quantitative differences between oxide and normal metal junctions. Whereas the oxide barrier is typically $10 \AA$ thick, a copper barrier might be $10,000 \AA$ or more. A typical resistance for an oxide junction is $1 \Omega$ but for a copper barrier, $10^{-6} \Omega$ or less. If we assume a critical current of $1 \mathrm{~mA}$, then the voltages developed when the critical value is exceeded would be $10^{-3} \mathrm{~V}$ and $10^{-9} \mathrm{~V}$ for an oxide and metallic barrier respectively. Thus the SNS junctions require low-voltage instrumentation for their study.

3. Experimental Results for Lead-Copper-Lead Junctions. - Junctions were made by evaporating successively into a glass slide a strip of lead, a disk of copper and a second strip of lead at right angles to the first. The lead strips were $0.2 \mathrm{~mm}$ wide and $1 \mu$ thick. The $i-V$ characteristics were measured by passing a current between the lead strips and measuring the resultant current with a null-reading superconducting voltmeter. The voltmeter consisted of a Slug with a resistance of about $10^{-7} \Omega$ in series to give at voltage resolution of about $10^{-14} \mathrm{~V}$.

A typical $i-V$ characteristic is shown in figure 6 . At sub-critical currents, no voltage appears across the junction; as the critical current is exceeded, there is a

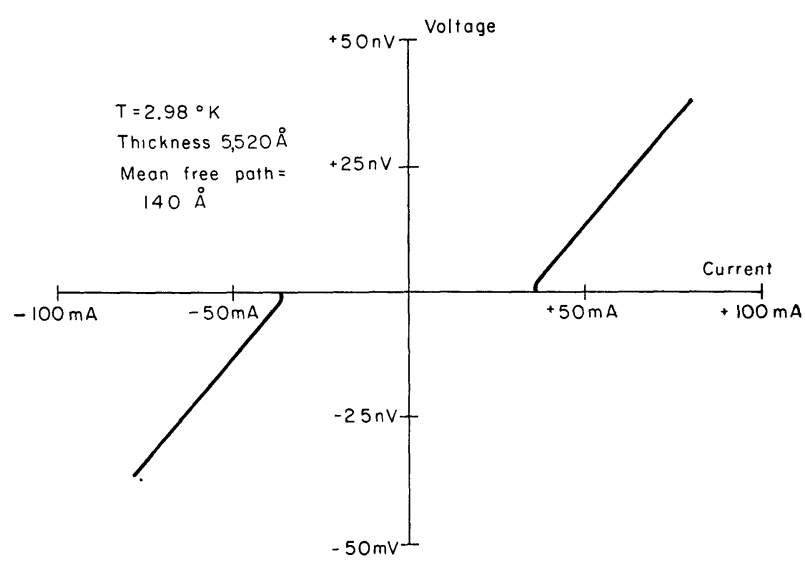

FIG. 6. $-i-V$ characteristic of SNS junction.

smooth rise in voltage which tends to a linear $i-V$ relationship. The differential resistance $\partial V / \partial i$ is essentially the resistance of the copper; the displacement of this characteristic from a parallel line through the origin can be explained in terms of a flux flow model.

A magnetic field applied in the plane at the junction modifies the critical current in the way indicated in

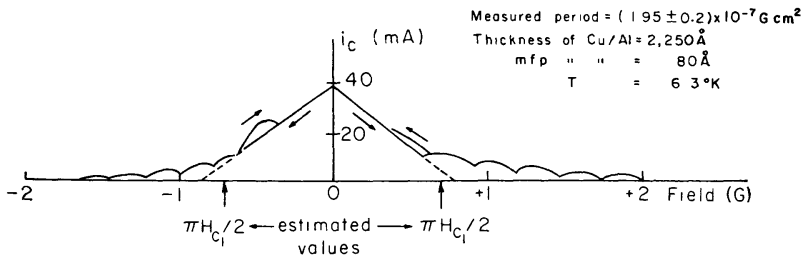

FIG. 7. $-i_{\mathrm{c}}$ against $H$ for SNS junction.

figure 7. The linear region at low fields is simply the Meissner effect while the oscillating behaviour at higher fields is essentially a Fraunhofer diffraction pattern. This behaviour very strongly indicates that these junctions exhibit the Josephson effect and that equation (3) is applicable.

It may be shown $[6,7]$ that the critical current of an SNS junction is of the form :

$$
i_{\mathbf{c}}(T)=A \frac{\xi_{N}(T)}{\xi_{s}^{2}(T)} \Delta_{s}^{2}(T) \exp \left(-2 a / \xi_{N}\right) .
$$

$A$ is a constant, $\Delta_{s}$ the energy gap of the superconductor and $2 a$ the barrier thickness. Notice that because of the exponential dependerice of $i_{\mathrm{c}}$ upon $T$ through $\xi_{N}(T)$, the critical current rises rapidly as $T$ is lowered. This may be contrasted with an insulating barrier for which $i_{\mathrm{c}}$ tends to a constant as $T$ is lowered. The equation can be conveniently analysed in three ways :

1) Near $T_{\mathrm{c} s}$, both $\Delta_{s}(T)$ and $\xi_{s}^{-1}(T)$ vary as $(1-t)^{\mathbf{1 / 2}}$ whereas $\xi_{N}(T)$ is essentially constant. Thus :

$$
i_{\mathrm{c}}(T) \simeq B(1-t)^{2}
$$

for $T \lesssim T_{\text {es }}$. This is in marked contrast to an oxide junction for which $i_{\mathrm{c}}(T) \simeq B^{\prime}(1-t)$.

2) For $T \lesssim T_{\mathrm{cs}} / 2$, both $\Delta_{s}(T)$ and $\xi_{s}(T)$ are essentially constant. For constant mean free path in the copper, we therefore find :

$$
i_{\mathrm{c}}(T) \simeq C \exp \left(-M T^{\mathbf{1} / 2}\right)
$$

for $T \lesssim T_{\mathrm{cs}} / 2$.

3) At all temperatures, and for constant m.f.p. in the copper :

$$
i_{\mathrm{c}}(T) \simeq D \exp (-N a) .
$$

Equations (6), (7) and (8) are in good agreement with experiment [7]. The behaviour of the junctions is thus relatively well understood.

4. Devige Application of SNS Junctions. - These junctions have several features which make them attractive as devices. Firstly, they may be fabricated in an evaporator or sputtering rig with a relatively high degree of reproducibility, particularly if alloys are used rather than pure metals. Further, these junctions may be thermally cycled between room and helium temperatures without undue deterioration. Their very low resistance $\left(<10^{-6} \Omega\right)$ means that the Johnson noise generated in the barrier is very small, so that the ultimate sensitivity of the junctions is probably higher than that of other types. As pointed out in I $(\S 3)$, external noise often dominates the junction noise unless adequate shielding is provided. The low impedance presented by the SNS junction is useful in this respect because the impedance mis-match to the 
external circuity is extremely high and consequently the amount of power radiated into the junction very small.

The overwhelming disadvantage of these junctions in device applications is obviously the very small voltages produced when the critical current is exceeded. It is clear that a second superconducting device is requi- red to detect the changes in critical current resulting from a signal applied to the SNS junction. Although the junction resistance could be increased somewhat by making the area smaller, it appears that these junctions are unlikely to become useful as devices except in applications where their advantages justify the complication of the superconducting instrumentation.

\section{REFERENCES}

[1] Josephison (B. D.), Phys. Letters, 1962, 1, 215; Rev. Mod. Phys., 1964, 36, 216; Adv. in Phys., 1965, 14, 419.

[2] JAKLEVic (R. C.), LAMbe (J.), Silver (A. H.) and MerCereau (J. E.), Phys. Rev. Letters, 1964, 12, 159.

[3] Clarke (J.), Phil. Mag., 1966, 13, 115 ; New Scientist, 1966, 29, 611; Proc. Symposium on the Physics of Superconducting Devices, Charlottesville, published by the Office of Naval Research, 1967.
[4] MCWane (J. W.), Neighbor (J. E.) and NeWBOWER (R. S.), Rev. Sci. Instr., 1966, 37, 1602.

[5] ZYCH (D. A.), Rev. Sci. Instr., 1968, 39, 1058.

[6] DE Gennes (P. G.), Rev. Mod. Phys., 1964, 36, 225; Superconductivity of metals and alloys, Benjamin (W. A.), New York, 1966.

[7] Clarke (J.), J. Physique, Colloque C2, Suppl. 2-3, 1968, 29, 3. Proc. Roy. Soc., 1969, A 308, 447. 